\title{
Robust allocation rules in dynamical cooperative TU Games
}

D. Bauso

\author{
P. V. Reddy
}

\begin{abstract}
Robust dynamic coalitional TU games are repeated TU games where the values of the coalitions are unknown but bounded variables. We set up the game supposing that the Game Designer uses a vague measure of the extra reward that each coalition has received up to the current time to re-adjust the allocations among the players. As main result, we provide a constructive method for designing allocation rules that converge to the core of the average game. Both the set up and the solution approach also provide an insight on commonalities between coalitional games and stability theory.
\end{abstract}

\section{INTRODUCTION}

This paper deals with robustness and dynamics in coalitional TU games along the line of [3], [4]. The above two elements naturally arise in all the situations where the coalitions values are uncertain and time-varying.

Robustness has to do with modeling coalitions' values as unknown entities. This is in spirit with some literature on stochastic coalitional games [14], [15]. However, we deviate from this stochastic framework since we model coalitions values as Unknown But Bounded variables within a priori known polytopic sets [5]. This has much in common with the recent literature on interval valued games [1], where the authors use intervals to describe coalitions values similarly to what is done in this paper. We also recognize some differences in that we focus more on the time-varying nature of the coalitions values. In doing this, we also link the approach to Set invariance Theory [6] which provides us some "nice" tools for stability analysis (see, e.g., the resort to a Lyapunov function in the proof of Theorem IV.1).

Dynamics enters into the picture in the form of a system state time evolution. The state accounts for the accumulated discrepancy between coalitions' values and allocations up to the current time with the assumption that the game is played repeatedly and continuously over time. So, the state accounts for the extra reward that a coalition has received up to the current time. At each time, different coalitions' values realize which are undisclosed to the Game Designer (GD) who then adjust allocations based on partial information on system state. Bringing dynamical aspects into the framework of coalitional TU games is an element in common with other papers [8], [10]. The main difference with those works is that the values of coalitions are realized exogenously and no relation exists between consecutive samples.

With this premise in mind, we are interested in providing certain convergence properties for the average game. Convergence conditions together with the idea that allocation rules

Dip. di Ingegneria Informatica, Università di Palermo, Viale delle Scienze, I-90128 Palermo, Italy, dario.bauso@unipa.it

Department of Econometrics and Operations Research, Tilburg University, The Netherlands, Email: P.V.Reddy@uvt.nl use a measure of the extra reward that a coalition has received up to the current time by re-distributing the budget among the players are a main issue in a number of other papers [2], [7], [9], [12], [13] as well. However, this paper departs from the aforementioned contributions mainly in that dynamics is there captured by a bargaining mechanism with fixed coalitions' values while we let the values be time-varying and uncertain. This last element adds some robustness in our allocation rule which have not been dealt with before.

The main result of the paper is a constructive method to design allocation rules that converge to the core of the average game. In addition to this, the set up of the game together with the proposed solution method put an accent on the sharing of common attributes between coalitional games and stability theory.

This paper is organized as follows. In Section II, we formulate the problem pointing out all issues that are high in our list of concern and make the problem challenging. In Section III, we present the basic idea of our solution approach which consists in turning the problem into a flow control one. In Section IV, we state the main result of this work regarding some convergence properties that come with a proper allocation rule design. In Section V, we provide some numerical illustrations. Finally, in Section VI, we draw some concluding remarks.

\section{Problem FORMULATION}

Consider a set of players $N=\{1, \ldots, n\}$ and all possible coalitions $S \subseteq N$ arising among these players. Introduce a time-varying characteristic function $\psi(S, t)$ which assigns a real value to each coalition $S$ at time $t \geq 0$ :

$$
\psi: 2^{N} \backslash\{\emptyset\} \times \mathbb{R}_{+} \rightarrow \mathbb{R}
$$

If we denote by $m=2^{n}-1$ the number of possible coalitions, we can view the characteristic function $\psi(., t)$ as returning a continuous-time signal in the $m$-dimensional space:

$$
v(t) \in \mathbb{R}^{m}, \quad \forall t \geq 0 .
$$

Turning from a function to a signal is useful to define the following dynamical coalitional games.

Definition II.1 (dynamical TU game) For each time $t \geq 0$, the instantaneous, integral, and average dynamical game $i s$ defined by the pairwise

- (instantaneous game) $\langle N, v(t)\rangle$, with $v(t) \in \mathbb{R}^{m}$;

- (integral game) $<N, \tilde{v}(t)>$, with $\tilde{v}(t)=\int_{0}^{t} v(\tau) d \tau$;

- (average game $)<N, \bar{v}(t)\rangle$, with $\bar{v}(t)=\frac{\tilde{v}(t)}{t}$. 
Henceforth, we use the symbol $\tilde{\psi}(t)$ and $\bar{\psi}(t)$ to indicate the integral and average up to time $t$ respectively of any given function $\psi(t)$. Also, the underlying assumption throughout this paper is that $v(t)$ is unknown to the GD but confined within a convex set at any time. We also assume that $v(t)$ is a mean ergodic stochastic process.

Assumption 1 (UBB and mean ergodic) Signal $v(t)$ is $U B B$ within a given convex set $\mathscr{V}: v(t) \in \mathscr{V} \in \mathbb{R}^{m}$. Furthermore, the expected value of $v(t)$ coincides with the long term average, i.e., $E[v(t)]=\lim _{t \rightarrow \infty} \bar{v}(t)$.

Under the above assumption, the core of the instantaneous game can be empty at some time $t$. Even if the above is true, we can still suppose that the core of the average game is non empty on the long run.

Assumption 2 (balancedness) The core of the average game is non empty in the limit: $\lim _{t \rightarrow \infty} C(\tilde{v}(t)) \neq \emptyset$.

We can view the above assumption as introducing some steady-state (average) conditions on a game scenario subject to instantaneous fluctuations.

Now, assume that the GD can take actions in terms of instantaneous allocations denoted by $a(t) \in \mathbb{R}^{n}$ and suppose the following budget constraints.

Assumption 3 (bounded allocation) The instantaneous allocation is bounded within a hyperbox in $\mathbb{R}^{n}$

$$
a(t) \in \mathscr{A}:=\left\{a \in \mathbb{R}^{n}: a_{\text {min }} \leq a \leq a_{\text {max }}\right\},
$$

with apriori given lower and upper bounds $a_{\min }, a_{\max } \in \mathbb{R}^{n}$.

Let us turn to comment on the information structure of the problem. To do this, we need to introduce some new terminology which is useful to clarify the information available to the GD.

For any coalition $S \subseteq N$, we define excess (extra reward) at time $t \geq 0$ as the difference between the total integral reward, given to it, and the integral value of the coalition itself, i.e.,

$$
\varepsilon_{S}(t)=\sum_{i \in S} \tilde{a}_{i}(t)-\tilde{v}_{S}(t)
$$

Furthermore, we say that $S$ is in excess at time $t \geq 0$ if the excess is non negative, i.e., $\sum_{i \in S} \tilde{a}_{i}(t) \geq \tilde{v}_{S}(t)$. In one word, coalitions in excess are those with respect to which the grand coalition of the integral game is stable. With the above clarification in mind, we henceforth assume that the GD has access to the limit of the average coalitions' values and to the vector of coalitions' excess $\varepsilon(t):=\left[\varepsilon_{S}(t)\right]_{S \subseteq N} \in \mathbb{R}^{m}$.

Assumption 4 (partial information) The GD knows $\lim _{t \rightarrow \infty} \bar{v}(t)$ and $\varepsilon(t)$ at each time $t \geq 0$. Furthermore, signal $v(t)$ and excess $\varepsilon(t)$ are non correlated.

The problem of interest consists in finding an allocation rule based on the available partial information so that if the instantaneous allocation is selected from this rule then the

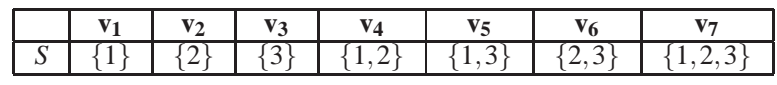

TABLE I

CORRESPONDENCES VERTICES-COALITIONS

average allocation converges to the core of the average game on the long run.

Problem 1 Find an allocation rule $f: \mathbb{R}^{m} \rightarrow \mathscr{A} \in \mathbb{R}^{n}$, such that if $a(t)=f(\varepsilon(t))$ then $\lim _{t \rightarrow \infty} \bar{a}(t) \in C(\bar{v}(t))$.

Remark II.1 (on relaxing information) In solving the problem, we will point out that it is not even necessary for the $G D$ to know $\varepsilon(t)$ as long as an oracle can tell him whether $\varepsilon(t)$ is greater or not than an opportune threshold given by the GD himself.

\section{A. Some comments on the above problem}

This section provides an alternative perspective on the problem in terms of controlling the topology of a hypergraph. To clarify more on this point, observe the implications that a given allocation may have on the dynamical coalitions scenario at any time $t \geq 0$. As an example, suppose that at each time $t \geq 0$ a certain number of coalitions naturally arise, say it for instance, $P, Q$ and $N \backslash(P \cup Q)$ and take the grand coalition as the ideal scenario. A well known fact is that we can use hypergraphs to describe coalitions scenario. Specifically, we can introduce a time-varying matrix $B(t)=$ $\left[\begin{array}{lll}c_{P} & c_{Q} & 1-\left(c_{P}+c_{Q}\right)\end{array}\right]$ where $c_{S}$ is the characteristic vector of the generic coalition $S \subseteq N$ and introduce $B_{\text {ref }}:=1 \in$ $\mathbb{R}^{n}$ as a reference topology. Note that $B(t)$ is nothing but the incidence matrix of the hypergraph describing a given coalitions scenario at time $t$. Now, if the average allocation is in the core of the average game, all coalitions are in excess, the grand coalition is stable with respect to all sub coalitions and the incidence matrix of the hypergraph is $B(t)=B_{\text {ref }}$. Viceversa, if $B(t) \neq 1 \in \mathbb{R}^{n}$, then some coalitions may be in excess some others not and it makes sense to adjust the allocation in order to drive $B(t)$ towards $B_{\text {ref }}$. Evidently, the reference topology could be any generic topology and not necessarily $1 \in \mathbb{R}^{n}$ and this enables us to generalize the reasoning.

\section{FLOW TRANSFORMATION}

The basic idea of our solution approach is to turn the problem into a flow control one. To do this, consider the hypergraph $\mathscr{H}$ with vertex set $V$ and edgeset $E$ as:

$$
\mathscr{H}:=\{V, E\}, \quad V=\left\{\mathbf{v}_{1}, \ldots, \mathbf{v}_{m}\right\}, \quad E:=\left\{\mathbf{e}_{1}, \ldots, \mathbf{e}_{n}\right\} .
$$

The vertex set $V$ has one vertex per each coalition whereas the edge set $E$ has one edge per each player.

A generic edge $i$ is incident to a vertex $\mathbf{v}_{j}$ if the player $i$ is in the coalition associated to $\mathbf{v}_{j}$. So, incidence relations are described by matrix $B_{\mathscr{H}}=\left[c_{S}^{T}\right]_{S \subseteq N} \in \mathbb{R}^{m \times n}$ whose rows are the characteristic vectors of all coalitions $S \subseteq N$. 


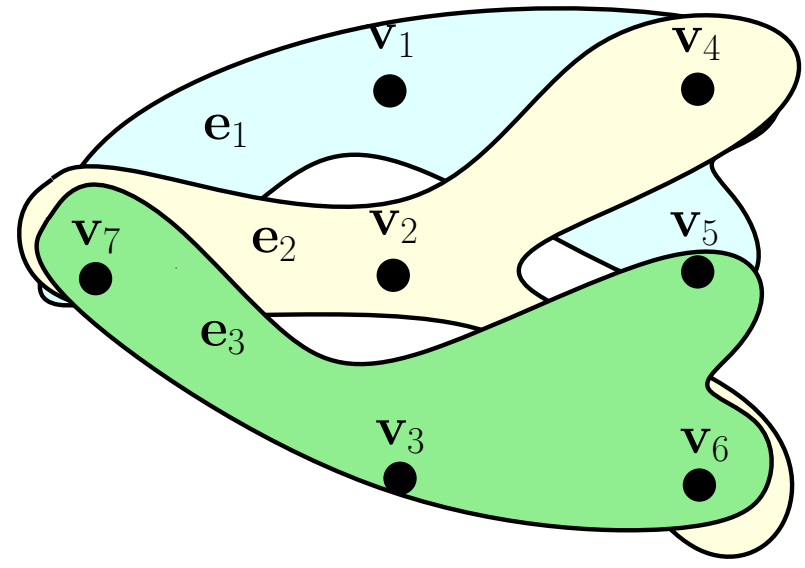

Fig. 1. Hypergraph $\mathscr{H}:=\{V, E\}$ for a 3-player coalitional game.

The flow control reformulation arises naturally if we view allocation $a_{i}(t)$ as the flow on edge $\mathbf{e}_{i}$ and the coalition value $v_{S}(t)$ of a generic coalition $S$ as the demand $d_{j}(t)$ in the corresponding vertex $\mathbf{v}_{j}$, namely $v_{S}(t)=d_{j}(t)$.

In view of this, allocation in the core translates into satisfying in excess the demand at the vertices. Specifically,

$$
\tilde{a}(t) \in C(\tilde{v}(t)) \quad \Leftrightarrow \quad B_{\mathscr{H}} \tilde{a}(t) \geq \tilde{d}(t)
$$

Now, since $\tilde{d}(t)$ is unknown at time $t$, we need to introduce some error dynamics which accounts for the derivatives of excesses:

$$
\dot{\varepsilon}(t)=B_{\mathscr{H}} a(t)-d(t), \quad d(t) \in \mathscr{V} .
$$

With the above in mind, the problem can be turned into a flow control problem where a controller wishes to drive the error $\varepsilon(t)$ (the excesses) to a target set

$$
\mathscr{T}:=\left\{\varepsilon \in \mathbb{R}^{m}: \varepsilon_{m}=0, \varepsilon_{j} \geq 0, \forall j=1, \ldots, m-1\right\} .
$$

But we can do more than this to simplify the tractability of

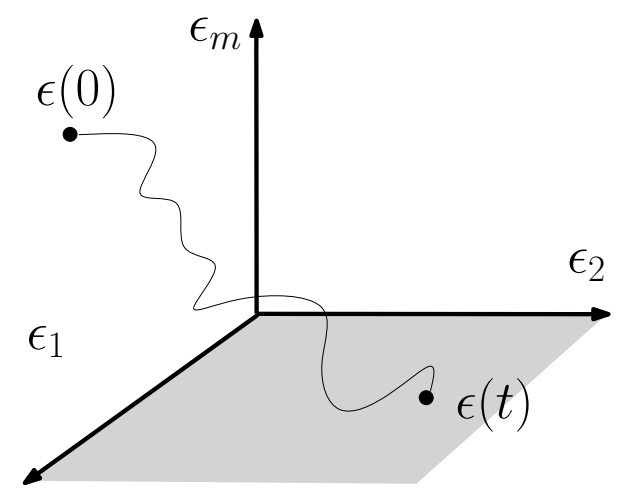

Fig. 2. Trajectory for $\varepsilon(t)$.

the problem. Using standard LP techniques we can introduce $m-1$ surplus variables (one per each coalition other than the grand coalition) so to project the allocation space into a one of higher dimension. In particular, let us expand control $u(t)=\left[a(t)^{T} \mid s(t)^{T}\right]^{T} \in \mathbb{R}^{(n+m-1)}$. This technique has the advantage of turning the inequalities (1) into equalities of type (see, e.g., [3], [4]):

$$
\dot{x}(t)=B u(t)-d(t), \quad d(t) \in \mathscr{V},
$$

where matrix $B$ is defined as

$$
B=\left[\begin{array}{c|c}
B_{\mathscr{H}} & -I \\
0
\end{array}\right] \in \mathbb{R}^{m \times(n+m-1)}
$$

and $I$ is an identity matrix of compatible dimensions. Variable $x(t) \in \mathbb{R}^{m}$ is now the state of the system. We still have to introduce the feasible controls set

$$
U:=\left\{u \in \mathbb{R}^{n+m-1}: a \in \mathscr{A}, s \geq 0\right\} \in \mathbb{R}^{n+m-1}
$$

and so the problem can be rephrased as follows: find a control strategy $\phi: \mathbb{R}^{m} \rightarrow U$ which drives the state $x(t)$ to zero in probability:

$$
u(t):=\phi(x(t)) \in U \quad \Leftrightarrow \quad \lim _{t \rightarrow \infty} x(t)=0
$$

with probability one. To tell it differently, we are requiring that dynamics $\dot{x}(t)=B \phi(x(t))-d(t)$ converge to zero in probability, or which is the same, we look for the stochastic stability of state $x(t)$.

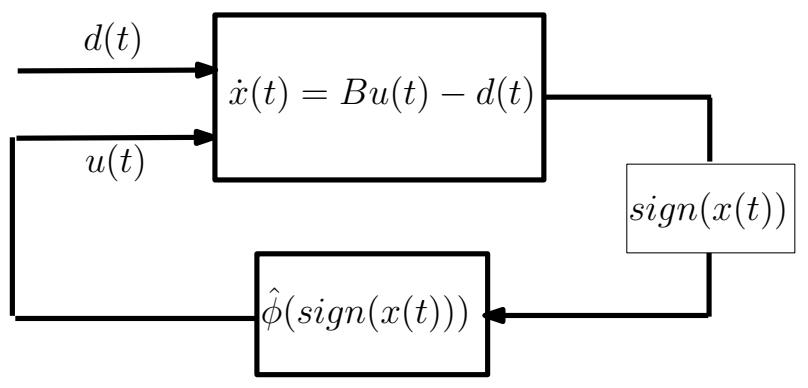

Fig. 3. Dynamical system.

\section{Stochastic Stability}

In this section, we state the main result of this work which proposes a solution to Problem 1 with information structure as discussed in Remark II.1. To do this, denote by $B^{\dagger}$ a generic pseudo inverse matrix of $B$ and take a feasible allocation $u_{\text {nom }}$ such that

$$
B u_{\text {nom }}=v_{\text {nom }}:=\lim _{t \rightarrow \infty} \bar{v}(t), \quad u_{\text {nom }} \in U .
$$

Also, for future purposes, define a function $\hat{\phi}($.$) , which$ depends only on the sign of $x(t)$, as follows:

$$
\hat{\phi}(\operatorname{sign}(x(t))):=u_{n o m}+\Delta u \in U, \quad \Delta u=-\delta B^{\dagger} \operatorname{sign}(x) .
$$

Now, taking the control $u(t)=\hat{\phi}(\operatorname{sign}(x(t)))$, we obtain the dynamic system $\dot{x}(t)=B \hat{\phi}(\operatorname{sign}(x(t)))-d(t)$ as displayed in Fig. 3. Now, we state the following convergence property.

Theorem IV.1 Dynamic system (4) with $\hat{\phi}(\operatorname{sign}(x(t)))$ as in (3) converges to zero in probability:

$$
\dot{x}(t)=B \hat{\phi}(\operatorname{sign}(x(t)))-d(t) .
$$


Proof: Consider a candidate Lyapunov function $V(x(t))=\frac{1}{2} x^{T}(t) x(t)$. The idea is to inspect that $E[\dot{V}(x(t))]<$ 0 for all $t \geq 0$. For this last to be true, it must be

$$
\begin{aligned}
E[\dot{V}(x(t))] & =E\left[x^{T}(t) \dot{x}(t)\right] \\
& =E\left[x^{T}(t) B u(t)\right]-E\left[x^{T}(t) v(t)\right] \\
& =E\left[x^{T}(t) B u_{\text {nom }}(t)\right]+E\left[x^{T}(t) B \Delta u(t)\right] \\
& -E\left[x^{T}(t) v_{\text {nom }}(t)\right]-\underbrace{E\left[x^{T}(t) \Delta v(t)\right]}_{=0} \\
& =E\left[x^{T}(t) B \Delta u(t)\right]<0 .
\end{aligned}
$$

where condition $E\left[x^{T}(t) \Delta v(t)\right]=0$ is a direct consequence of Assumption 4. But the above condition $E\left[x^{T}(t) B \Delta u(t)\right]<0$ is satisfied since $B \Delta u(t)=-\delta \operatorname{sign}(x)$, which in turn implies

$$
E\left[x^{T}(t) B \Delta u(t)\right]=E\left[-\delta\|x(t)\|_{1}\right]<0 .
$$

Corollary IV.1 The average allocation converges to the core of the average game:

$$
\lim _{t \rightarrow \infty} \bar{a}(t) \in C(\bar{v}(t)) .
$$

Proof: For the above condition to be true it suffices to prove i) $B \bar{u}=\bar{v}$ and ii) $\bar{u} \in U$. Proving ii) is straightforward from $u(t)=\hat{\phi}(\operatorname{sign}(x(t))) \in U$ for all $t \geq 0$. Condition i) is a direct consequence of Theorem IV.1. Actually convergence in probability of (4) to zero means $\lim _{t \rightarrow \infty} \bar{x}(t)=0$ and this in turn implies $B \bar{u}=\bar{v}$.

\section{A. Comments on available information}

In this subsection we expand more on the information structure of the problem. In particular, we highlight how the whole feedback on state $x(t)$ can be reviewed as the result of an oracle-based procedure.

To see this, let us start by recalling that the control strategy is based on the only sign of state $x(t)$ which can be expressed by

$$
x(t)=B \tilde{u}(t)-\tilde{d}(t)=\varepsilon(t)-\tilde{s}(t) .
$$

The above condition relates state $x(t)$ to the excess $\varepsilon(t)$. Now, with reference to component $j$, the sign of $x_{j}(t)$ yields:

$$
\operatorname{sign}\left(x_{j}(t)\right):=\left\{\begin{array}{cc}
1 & \varepsilon_{j}(t)>\tilde{s}_{j}(t) \\
0 & \varepsilon_{j}(t)=\tilde{s}_{j}(t) \\
-1 & \varepsilon_{j}(t)<\tilde{s}_{j}(t) .
\end{array}\right.
$$

In the above condition, review $\tilde{s}_{j}(t)$ as a nonnegative threshold and notice that "the GD has access to the sign of $x_{j}(t)$ " has the following interpretation: after submitting such a threshold to an oracle, the GD is revealed whether the excess exceeds such threshold or not. Recall that nonnegativeness of the threshold has its roots in the feasibility condition $u(t) \in U$ for all $t \geq 0$ with feasible set $U$ as in (2).

Nonnegativeness of the threshold provides us with a further comment on the information available to the GD. Actually, from the first condition in (6), we can conclude that coalitions associated to a positive state $x(t)$ are certainly in excess. This is clear if we observe that $\operatorname{sign}\left(x_{j}(t)\right)=$
1 implies $\varepsilon_{j}(t)>\tilde{s}_{j}(t) \geq 0$. We can then summurize the information content available to the GD as follows, let $S$ be the generic coalition associated to component $j$ :

$$
\operatorname{sign}\left(x_{j}(t)\right):=\left\{\begin{array}{cl}
1 & \text { then coalition } S \text { in excess } \\
-1,0 & \text { nothing can be said. }
\end{array}\right.
$$

Trivially, the present developement, which is all based on control strategy (3), fits the case where $x(t)$ is revealed completely as abundantly elaborated in [4]. In this last case, the fact that the GD knows $x(t)$ implies from (5) that he knows $\varepsilon(t)$ as well. Also, it is intuitive to infer that in this last set up, exact knowledge of $x(t)$ can only influence positively the GD in terms of speed of convergence of allocations into the core of the average game. All the above comments go back and try to clarify what was anticipated in Remark II.1 where it was stated that it is not even necessary that the GD knows exactly $\varepsilon(t)$.

\section{NUMERICAL ILLUSTRATIONS}

Consider a 3 player cooperative game, so $m=7$, with the following intervals for values of coalitions:

$$
\begin{aligned}
& v(\{1\}) \in[0,4], v(\{2\}) \in[0,4], v(\{3\}) \in[0,4], \\
& v(\{1,2\}) \in[0,4], v(\{1,3\}) \in[0,6], \\
& v(\{2,3\}) \in[0,7], v(\{1,2,3\}) \in[0,12] .
\end{aligned}
$$

The convex set $\mathscr{V}$ is characterized by the above intervals. From Assumption 4 GD knows the long run average game, i.e., $\lim _{t \rightarrow \infty} \bar{v}(t)=v_{\text {nom }}$. We consider the choice of balanced nominal game as $v_{\text {nom }}=\left[\begin{array}{lllllll}1 & 2 & 3 & 4 & 5 & 6 & 10\end{array}\right]^{T}$. During simulations the instantaneous games $v(t) \in \mathscr{V}$ should satisfy the average behavior given by:

$$
\lim _{t \rightarrow \infty} \frac{1}{t} \int_{0}^{t} v(\tau) d \tau=v_{\text {nom }}
$$

Next, we describe an algorithm to generate $v(t) \in \mathscr{V}$ such that the above condition holds true.

\section{Algorithm:}

1) Generate $m$ random points, $r_{i} \in \mathscr{V} \subset \mathbb{R}^{m}, i=1,2, \cdots, m$.

2) Solve $R \cdot p=v_{n o m}$, with $R=\left[r_{1}, r_{2}, \cdots r_{m}\right]$.

3) If $p \geq 0$ and $\mathbf{1}^{T} p>0$, then go to (4) else go to (1).

4) Rescale $R$ as $R=\left(\mathbf{1}^{T} p\right) R$ and $p$ as $p=\frac{p}{\left(\mathbf{1}^{T} p\right)}$

5) If $r_{i} \in \mathscr{V}, i=1,2, \cdots, m$, then go to (6) else go to (1).

6) STOP

By construction, of the algorithm, $v_{\text {nom }}$ is in the relative interior of the convex hull generated by columns of the matrix $R$. If an instance of the game $v(t)$ is chosen as $r_{i}$ with probability $p_{i}$ from the pair $(R, p)$, Assumption 4 is satisfied. For simulations we ran the algorithm 20 times to generate a total of 140 points (or $20(R, p)$ pairs) in $\mathscr{V}$. Further, from each of the 20 pairs we take 2000 random selections (using Matlab randsrc function), which amounts to 40,000 instantaneous games $v(t)$. The nominal choice of allocations and surplus is taken as $u_{\text {nom }}=\left[\begin{array}{lllllllll}2.5 & 3 & 4.5 & 1.5 & 1 & 1.5 & 1.5 & 2 & 1.5\end{array}\right]^{T}$. It can be verified that $B u_{\text {nom }}=v_{\text {nom }}$. 
Choice of $\delta$ : For simulations, the choice of $\delta$ is crucial so as to ensure $u(t) \in U$. This condition translates to $U_{\min } \leq u_{\text {nom }}+\delta B^{\dagger} \operatorname{sign}(x) \leq U_{\max }$. We observe $-\sum_{j}\left|B_{i j}^{\dagger}\right| \leq\left(B^{\dagger} \operatorname{sign}(x)\right)_{i} \leq \sum_{j}\left|B_{i j}^{\dagger}\right|$. A conservative estimate of $\delta$ is obtained as $U_{\text {min }} \leq u_{\text {nom }} \pm \delta \max _{i}\left\{\sum_{j}\left|B_{i j}^{\dagger}\right|\right\} \leq U_{\text {max }}$. For $m=7$, we have $\max _{i}\left\{\sum_{j}\left|B_{i j}^{\dagger}\right|\right\}=2.11$. For the instantaneous game a negative allocation/surplus is not allowed, so $U_{\min } \geq 0$.1. Furthermore, an allocation/surplus greater than the value of grand coalition is not allowed, so $U_{\max } \leq v_{\text {nom }}(N)$.1. We chose $\delta=0.75$, which satisfies the above stated requirements. The robust allocation rule is implemented numerically with a stepsize of $\Delta=0.01$. Next, we present performance results of the robust control

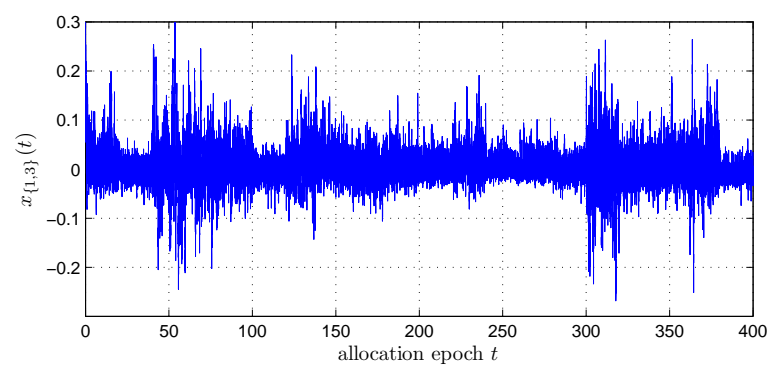

(a) Time plot of $x_{\{1,3\}}(t)$.

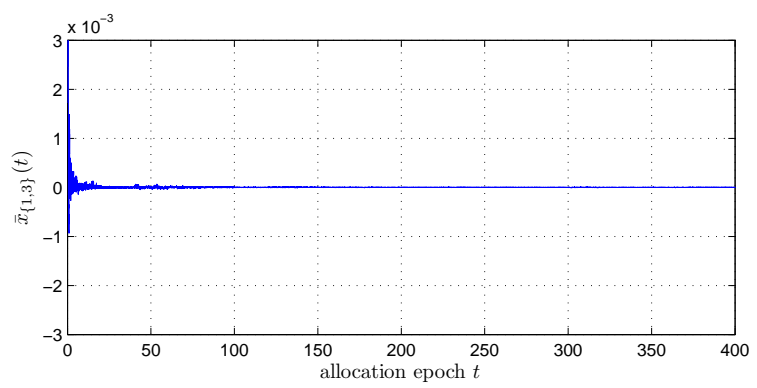

(b) Time plot of $\bar{x}_{\{1,3\}}(t)$.

Fig. 4. Performance of the robust control law $\hat{\phi}(\operatorname{sign}(x(t)))$.

law given by equation (3). From Theorem IV.1, $x(t)$ converges to zero in probability with a specific choice of control law. Fig. 4(a) illustrates this behavior (for coalition $\{1,3\})$. Further, by Corollary IV.1, the same control law ensures that the average game is balanced in a long run, in other words $\lim _{t \rightarrow \infty} \bar{x}(t)=0$ and Fig. 4(b) illustrates this behavior (for coalition $\{1,3\}$ ). The control law ensures $E[\dot{V}(x(t))]$ is negative for all $t>0$, we illustrate this behavior in Fig. 5. From Corollary IV.1, the average allocation vector is contained in the core of the average game, i.e. $\lim _{t \rightarrow \infty} \bar{a}(t) \in C(\bar{v}(t))$. However, the control law does not guarantee the convergence of allocation vectors to the nominal allocation vector on a long run. We illustrate this observation in Fig. 6.

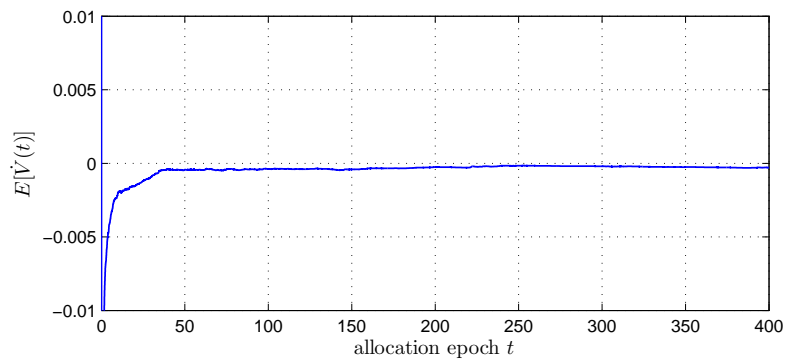

Fig. 5. Time plot of $E[\dot{V}(x(t))]$

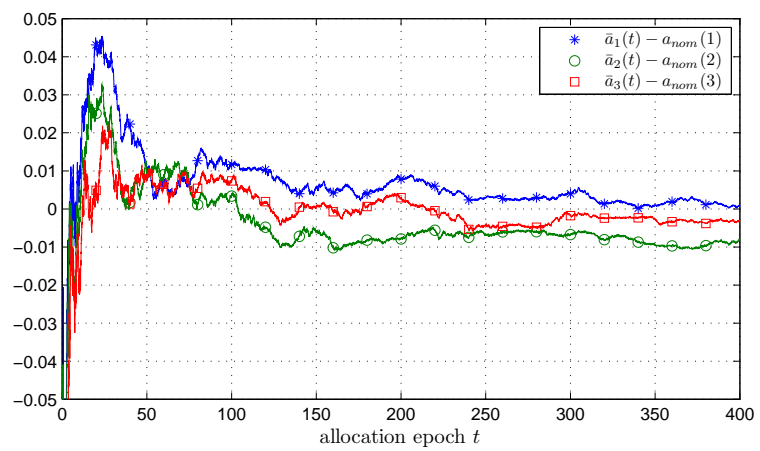

Fig. 6. Time plot of $\bar{a}(t)-a_{\text {nom }}$.

\section{CONCLUSIONS AND FUTURE WORK}

In this paper we derived a robust control law that ensures the average allocation vector is contained in the core of the average game. However, the control law is derived on the premise that GD knows apriori, the nominal allocation vector. If this information is not available the derivation of control law implicitly involves solving an LP problem. Currently this work is in progress. By further relaxing the information requirement, the problem can be treated as a learning process where the GD is trying to learn the nominal game from instantaneous games. We postpone working in this direction for the future.

\section{REFERENCES}

[1] S.Z. Alparslan Gök, S. Miquel, and S. Tijs, "Cooperation under interval uncertainty", Mathematical Methods of Operations Research, vol. 69, no. 1, 2009, pp. 99-109.

[2] T. Arnold., U. Schwalbe, "Dynamic coalition formation and the core", Journal of Economic Behavior and Organization, vol. 49, 2002, pp. 363-380.

[3] D. Bauso and J. Timmer, "Robust Dynamic Cooperative Games", International Journal of Game Theory, vol. 38, no. 1, 2009, pp. 23-36.

[4] D. Bauso, J. Timmer, "On robustness and dynamics in (un)balanced coalitional games", Memorandum 1916, Department of Applied Mathematics, University of Twente, 2010, ISSN 1874-4850.

[5] D. P. Bertsekas and I. B. Rhodes, "On the Minimax Reachability of Target Sets and Target Tubes", Automatica, vol. 7, pp. 233-241, March 1971.

[6] F. Blanchini, "Set invariance in control - a survey", Automatica, vol 35, no. 11, pp. 1747-1768, 1999.

[7] J.C. Cesco, "A convergent transfer scheme to the core of a TU-game", Revista de Matemáticas Aplicadas, vol. 19, no. 1-2, 1998, pp. 23-35.

[8] J.A. Filar and L.A. Petrosjan, "Dynamic Cooperative Games", International Game Theory Review vol. 2, no. 1, 2000, pp. 47-65. 
CONFIDENTIAL. Limited circulation. For review only.

[9] J. H. Grotte, "Dynamics of cooperative games", International Journal of Game Theory, vol. 5, no. 1, pp. 27-64.

[10] A. Haurie, "On some Properties of the Characteristic Function and the Core of a Multistage Game of Coalitions", IEEE Transactions on Automatic Control, vol. 20, no. 2, 1975, pp. 238-241.

[11] L. Kranich, A. Perea, H. Peters, "Core concepts in dynamic TU games", International Game Theory Review, vol. 7, 2005, pp. 43-61.

[12] E. Lehrer, "Allocation Processes in Cooperative Games", International Journal of Game Theory, vol. 31, 2002, pp. 341-351.

[13] A. Sengupta, K. Sengupta, "A property of the core", Games and Economic Behavior, vol. 12, 1996, pp. 266-273.

[14] J. Suijs and P. Borm, "Stochastic Cooperative Games: Superadditivity, Convexity, and Certainty Equivalents", Games and Economic Behavior, vol. 27, no. 2, 1999, pp. 331-345.

[15] J. Timmer, P. Borm, and S. Tijs, "On three Shapley-like solutions for cooperative games with random payoffs", International Journal of Game Theory, vol. 32, 2003, pp. 595-613. 